\title{
Apuntes para la revisión teórica de las TIC en el ámbito de la educación superior.
}

\section{Notes for the Theoretical Revision of ICT in the Field of Higher Education.}

\author{
Carlos Neri \\ carlosipc@gmail.com \\ Universidad de Buenos Aires. Argentina. \\ Diana Fernández Zalazar \\ dfzalazar@gmail.com \\ Universidad de Buenos Aires. Argentina.
}

\begin{abstract}
Resumen
Este artículo plantea, desde un punto de vista socio-constructivista, unas notas para la revisión teórica sobre lo avanzado en las bases del uso de la tecnología en la Educación Superior.

Se pone énfasis en generar una comunicación y elaboración de contenidos, reformulando las clases y convirtiéndolas en un ágora o foro en vivo a partir de las líneas de trabajo presentadas a distancia. Los docentes universitarios, sobre todo en las clases teóricas o denominadas magistrales, trabajamos durante 90, 180 e incluso 240 minutos un tema por clase. Sobre el final y pocas veces durante el transcurso de las mismas, los alumnos realizan preguntas cerrándose los intercambios posibles. Sabemos que cualquier profesor maneja una cantidad de conocimientos y una articulación de los mismos, que no necesariamente aparece en las clases teóricas más centradas en la presentación de un tema. El tiempo de las clases y su formato restringe el despliegue de contenidos y las líneas de trabajo que se abren quedan inconclusas. Proponemos entonces, dinamizar los prácticos y los teóricos, produciendo una inversión de la clase. La apuesta es presentar contenidos transmedia articulados al uso de las redes, que amplíen las distintas formas y posibilidades de apropiación y producción de conocimientos.
\end{abstract}

Palabras claves

TIC, Docencia, Educación, Socioconstructivismo.

\footnotetext{
Abstract

This article discusses, from a socio-constructivist perspective, some notes for the theoretical revision of the progress on the use of technology in higher education.

The emphasis resides in creating a communication and elaborations of the academic topics, reformulating the classes and turning them into an agora or forum where it is possible to work remotely.

During the master classes, university teachers talk about certain topics for about 90,180 or even 240 minutes. Question from students have place at end of the lecture, rarely during the same. Any possibility of knowledge exchange among teacher-student is limited to that moment.

Any professor possesses a much great knowledge and the possibilities of articulation among concepts that exceed the presentation of any topic.

The duration of the classes and its format restricts the display of the academics themes. We suggest a dynamic transformation of the practical and theorical classes in order to generate an inversion of those spaces.

The proposal is to present transmedia contents articulated with the use of networks, that way the possibilities of ownership and production of knowledge are greatly increased.

Key words

ICT, Teaching, Higher Education, Socioconstructivism
} 


\section{Introducción:}

Las tecnologías de la información y la comunicación (TIC) atraviesan el escenario de la vida universitaria, instalándose de diferente manera en los procesos de enseñanza y aprendizaje, así como en la relación de todos los actores de la comunidad académica. En su formas más elaboradas y actuales aparecen como: Entornos Personales de Aprendizaje (Personal Learning Enviroment, PLE) (Severance, Hardin, y Whyte, 2008), Tecnologías de Empoderamiento y Participación (TEP) (Dreig, 2012), y en los ambientes de colaboración (Bruffee, 1999), y en los denominados MOOC ( Massive Open Online Course). En todos los casos mencionados se requiere un cambio de perspectiva en la práctica docente, yendo más allá del sujetamiento a los usos instrumentales-mercantiles (Benbenaste, 2007) así como a prácticas heredadas de la docencia tradicional que repiten con tecnología lo que se hace sin ella.

Las prácticas de enseñanza a nivel universitario han sido poco estudiadas (CidSabucedo, Pérez-Abellás \& Zabalza, 2009), siendo la mayor parte de los trabajos e investigaciones realizados los referidos a la educación media, a la vez que la incorporación de las TICS en las prácticas docentes implican necesariamente un diseño tecno-pedagógico o tecno-instruccional que produce contenidos, objetivos y actividades de enseñanza y aprendizaje (Coll, 2009).

Sabemos que diferentes etapas se suceden al implementar tecnología en una institución así como las problemáticas que afectan a los docentes durante este proceso (Schibeci et al., 2008), el proyecto Acout (Ringstaff, Yocam, y Marsh, 1996); Adell, J. (2008). La descripción de las etapas en el proceso de apropiación ha surgido a partir de investigaciones sobre las prácticas docentes en niveles no universitarios, pero creemos que es posible pensarlas también en relación a la docencia universitaria, dado que la secuencia que plantea no es necesariamente rígida, pero a la vez se mencionan pasos a cumplir en este orden: 1- el acceso; 2- la adopción; 3- la adaptación; 4la apropiación y 5- la innovación.

Esta secuencia debe ser analizada a la luz de un enfoque contextualista, heredero de los postulados básicos de la teoría socio-histórica de Vigotsky y de las indagaciones sobre el aprendizaje y el desarrollo socialmente situado (Cole, 1999; Cole y Engeström, 2001; Chaiklin y Lave, 2001; Salomon, 2001). Destacando la importancia de los instrumentos de mediación, en tanto artefactos (Cole, 1999), que articulan procesos psicológicos con el uso de instrumentos de mediación cultural, donde se relacionan los sujetos en el contexto de las prácticas y en la interacción con los otros. También cabe la pregunta sobre las limitaciones que los determinantes duros de la educación ponen a las innovaciones educativas y ello como se refleja en las prácticas de enseñanza. Para abordar esta problemática entendemos que la misma se despliega en el marco de un sistema de actividad (Engeström, 2008), donde las relaciones entre docentes, alumnos, contenidos, normativas, comunidad, institución, etc. se deben analizar de manera interdependiente, dado que nos enfrentamos con un problema complejo, y desde la visión sistémica de la complejidad (García, 2011).

Apuntes para la revisión teórica de las TIC en el ámbito de la educación superior. Carlos Neri y Diana Fernández Zalazar. 


\section{Desarrollo:}

Analizaremos a partir de 4 ejes las posibilidades y limitaciones de las TIC en el ámbito académico.

\section{1.- Las funciones psicológicas superiores y su relación con las TIC.}

Desde los primeros estadios de la humanidad, la apoyatura del hombre en elementos externos muestran que siempre existió una apoyatura de la funciones psicológicas superiores y es desde la cultura que éstas, sean en sus formas rudimentarias como ayuda memorias con nudos en los dedos o hasta los ordenadores, se han amplificado y reformulado ampliando sus límites. De esta manera acordamos que: "en lo esencial, cuando se considera que la mediación a través de artefactos constituye la característica fundamental distintiva de los seres humanos, se está declarando que se adopta la idea de que la cognición humana está distribuida" (Cole \& Engeström, 2001, p. 69).

$\mathrm{Si}$ pensamos en la posibilidad de un reservorio de datos, como es la Web, y una interacción permanente con las tecnologías por parte de los jóvenes, podremos observar cambios en las funciones superiores alterándose fundamentalmente la memoria y la atención. Estos observables muestran otras competencias instrumentales en los alumnos, que requieren ser tomadas por el ámbito educativo y potenciadas para la producción de conocimientos. La cognición así se abre paso a modelos distribuidos.

Teniendo en cuenta los aportes de Vigotsky y de autores contemporáneos que trabajan desde la perspectiva de la cognición distribuida, como Cole y Engeström (2001), encontramos que se cuestionan a sí mismos preguntándose si tal modelo significa un avance respecto de la teoría sociocultural rusa, y se responden afirmando que en todo caso es un avance "leve". De alguna manera dichos desarrollos no hacen mucho más que enfatizar la "distribución extra somática de la cognición" (Cole y Engeström, 2001, p. 36). Gardner acuerda con este enfoque al sostener que "lejos de quedar limitadas al cráneo del individuo, la cognición y la inteligencia están distribuidas por el paisaje" (Gardner, 2008, p. 118). En el mismo sentido investigaciones sobre los buscadores como google son pensados como la memoria externa del sujeto y así encontramos: "Los resultados de cuatro estudios sugieren que cuando se enfrentan a preguntas difíciles, las personas están preparadas para pensar acerca de las computadoras y que cuando las personas esperan tener acceso en el futuro a la información, que tienen menores tasas de recuperación de la información en sí misma y la recuperación mejorada en lugar de dónde se puede acceder ella. El Internet se ha convertido en una forma primaria de memoria externa o transactivo, donde la información se almacena en conjunto fuera de nosotros mismos..." (Sparrow, Liu y Wegner, 2011).

La importancia de investigaciones de este tipo está dada por el impacto que se produce en el alumno, cuyo sistema educativo está organizado en función del recordar; mientras que en la esfera del mercado, el sujeto desplaza las funciones de la memoria al dispositivo que funciona como prótesis mental. Se recuerdan menos datos y se recuperan éstos desde los soportes externos, teléfonos y computadoras. Esta situación nos ubica en una de las tensiones entre modelo educativo y la cognición en la actualidad.

Apuntes para la revisión teórica de las TIC en el ámbito de la educación superior. Carlos Neri y Diana Fernández Zalazar. 


\section{2.- El problema del tiempo y la distribución en las clases magistrales}

En tiempos fluidos, tiempos "baumianos" de atención dividida, la organización de las clases teóricas o magistrales sufren una crisis silenciosa. Sostenidas en el prestigio del orador, titular o adjunto de una cátedra se organizaron históricamente como el espacio de reflexión teórica donde se "iluminaba" a la concurrencia sobre los avatares del campo disciplinar exponiéndose líneas teóricas y contradicciones. Con una duración entre dos a cuatro horas, heredan del púlpito, la transmisión del mundo del saber y la verdad revelada. Jorge Luis Borges en Ruinas Circulares nos acerca a una metáfora de este mundo:

"... El forastero se soñaba en el centro de un anfiteatro circular que era de algún modo el templo incendiado: nubes de alumnos taciturnos fatigaban las gradas; las caras de los últimos pendían a muchos siglos de distancia y a una altura estelar, pero eran del todo precisas. El hombre les dictaba lecciones de anatomía, de cosmografía, de magia: los rostros escuchaban con ansiedad y procuraban responder con entendimiento, como si adivinaran la importancia de aquel examen, que redimiría a uno de ellos de su condición de vana apariencia y lo interpolaría en el mundo real. El hombre, en el sueño y en la vigilia, consideraba las respuestas de sus fantasmas, no se dejaba embaucar por los impostores, adivinaba en ciertas perplejidades una inteligencia creciente. Buscaba un alma que mereciera participar en el universo..."

Sin embargo las "almas a ser elegidas", van encontrando otros caminos para su aprendizaje y este tipo de clase podríamos pensarlas afectadas por el concepto de “economía de la atención". Como señaló Herbert Simon (1971) en tiempos previos a la abundancia de datos en Internet, se refiere a cómo la riqueza de información produce una pobreza de atención. Esta tensión entre los datos y la capacidad de atender, en el escenario de una renovación permanente de la presentación de los mismos, debe dar lugar a un primer paso donde el aparato psíquico convierta los datos en información, relacionando lo nuevo con lo aprendido para luego llegar al paso siguiente, la producción de conocimiento. En tiempos de crisis de la atención, se aggiorna el modelo con algunas variantes como las presentaciones en ppt, Prezi o las pizarras electrónicas.

Como señala Restrepo (2005): "Pero aún más relevante para los propósitos de la educación, es la concepción sobre la naturaleza de los contenidos que subyace a la clase magistral, la de verdades concluidas. Las verdades son hechos establecidos, dentro de la perspectiva de que pertenecen a un mundo real de objetos incuestionables que tienen existencia propia e independiente, no solamente del estudiante, sino del profesor (von Glasersfeld citado en Phillips, 1995). Son transmitidas en forma vertical hacia unos alumnos cuyo papel es el de receptores pasivos, aislados y distantes. La concepción de aprendizaje en esta práctica está basada en la memoria (Carretero, 1993, p. 27). La misma disposición de los ambientes de clase alineados en filas y columnas que tienen como su centro de atención al profesor refleja estas concepciones (Koop, Stanford, Rohlfing y Kendall, 2004)”

En relación a la modernización por el uso de las TIC, Area Moreira (2000), pone el foco en las problemáticas a cambiar: El reto de futuro está en que las universidades innoven no sólo su tecnología, sino también sus concepciones y prácticas pedagógicas, lo que significa modificar el modelo de enseñanza universitario en su globalidad. Abordar este

Apuntes para la revisión teórica de las TIC en el ámbito de la educación superior. Carlos Neri y Diana Fernández Zalazar. 
proceso significará reformular el papel y práctica pedagógica del docente, planificar y desarrollar modelos de aprendizaje del alumnado radicalmente distintos a los tradicionales, cambiar las formas organizativas del tiempo y el espacio de las clases, cambiar las modalidades y estrategias de tutorización..."

\section{3.- Las Narrativas transmediales.}

Las narrativas transmedia son un modo de apropiación cultural en la que muchos jóvenes están interactuando a nivel de las producciones del mercado. El término narrativa transmedia se define como: "La narración transmediática se refiere a una nueva estética que ha surgido en respuesta a la convergencia de los medios, que plantea nuevas exigencias a los consumidores y depende de la participación activa de las comunidades de conocimientos. La narración transmediática es el arte de crear mundos. Para experimentar plenamente cualquier mundo de ficción, los consumidores deben asumir el papel de cazadores y recolectores, persiguiendo fragmentos de la historia a través de canales mediáticos, intercambiando impresiones con los demás mediante grupos de discusión virtual, y colaborando para garantizar que todo aquel que invierta tiempo y esfuerzo logre una experiencia de entretenimiento más rica...." (Jenkins, 2008).

La lógica de las narrativas transmediales puede pensarse en educación como situaciones de inmersión del alumno en el conocimiento, narrando en diferentes soportes que le son tanto o más familiares que la oralidad lineal de las exposiciones o la lectoescritura tradicional. Como bien ha observado Carlos Scolari (2012): "Para evitar una intoxicación semántica lo mejor es pensar al transmedia como un tipo muy específico de producción cultural o discurso donde el relato: a) se expande a través de varios medios y plataformas, y b) los "usuarios" participan en esa expansión...". Podemos pensar en el campo educativo una situación donde se conjugue la producción y apropiación de conocimiento en un ambiente transmedial y colaborativo de cogniciones distribuidas.

\section{4.- El concepto de clase invertida}

En el año 2007, Jonathan Bergman y Aarom Sams del instituto Woodland Park trabajan sobre un software que combina presentaciones en power point con videos, en ese momento llamaron a su sitio Educational Vodcasting, lo que llevó a la confusión de que el modelo planteado se reducía a la instrucción a través de videos para ser vistos en la casa, sin tomar en cuenta que se dejaba abierto a diversas aplicaciones de screencasting. Finalmente, el término The Flipped Classroom, quedó acuñado en el 2011 en el nombre de su libro: "Flip Your Classroom: Reach Every Student in Every Class Every Day" (Sams,

2011).

Muchos recursos se han agregado al concepto de clase tecnológica, pero todos bajo la misma lógica, los aprendizajes fuera del espacio áulico y el retorno a este para avanzar sobre lo aprendido desde otro lugar, más allá de la denominada clase magistral. En este tipo de modelo se aprovecha el tiempo presencial para avanzar con actividades de aprendizaje significativo y personalizado, estimulando los intercambios y debates, el desarrollo de proyectos, el análisis de casos, las prácticas de laboratorio; generando un entramado donde la dinámica del aula se convierta en actividad en red.

Apuntes para la revisión teórica de las TIC en el ámbito de la educación superior. Carlos Neri y Diana Fernández Zalazar. 
El lugar del docente también se redefine en este entorno, donde pasa a tener el rol de tutor o guía del proceso de aprendizaje, orientando al alumno en los contenidos, recorridos y estrategias que favorezcan el pensamiento crítico y los recursos metacognitivos en función de la construcción de conocimientos. Esto lleva a un cambio cultural donde la obligatoriedad a asistir a la exposición teórica queda sustituida por la actividad de los alumnos y el docente en el marco de un aprendizaje más flexible y autónomo.

\section{Conclusiones:}

Al articular los 4 ejes de esta exposición queda claro que las cogniciones en la actualidad funcionan de manera distribuida y facilitada por la flexibilidad que aporta la tecnología en diversos soportes y formatos. Queda repensar y trabajar sobre el núcleo duro de la educación que resiste a esta nueva configuración del espacio y del tiempo para pasar a una propuesta que enmarque los nuevos desarrollos del conocimiento con formas y herramientas acordes a los tiempos y la subjetividad de nuestros alumnos. Para ello nos interesa la posibilidad de producir objetos de aprendizaje que permitan "descargar" la carga cognitiva de la memoria y la atención. Si la transmisión de datos se da en un entorno extra áulico, el tiempo áulico puede ser repensado para la discusión y profundización con mediaciones fuertes que permitan la inclusión y el desarrollo de conocimiento.

La utilización de muros compartidos, twitter y otros recursos en la clase teórica, convertirán ésta no sólo en una exposición o profundización de los objetos de conocimiento trabajados fuera del aula, sino en una verdadera red social de distribución y producción de los mismos. Una red social que articulará lo presencial con lo virtual en una dinámica flexible y acorde a las particularidades del grupo y a la vez a la singularidad de cada proceso de aprendizaje. Esta producción en la clase teórica se convierte también en un objeto de aprendizaje, descargable por códigos QR o desde el sitio o aula virtual de la cátedra. Es decir, serán objetos reutilizables, pasibles de ser intercambiados por la comunidad de aprendizaje y asequible a todos aquellos que lo requieran. Un verdadero entramado en red donde la clase presencial se transmuta en una red social presencial-virtual que permitirá expandirse a través de esos objetos de aprendizaje en otras redes que colaborarán y enriquecerán lo producido, generando el efecto emergente de un sistema complejo que se autoorganiza en el proceso de aprendizaje.

Para ello la actividad de los sujetos en la red deberá ser orientada por los educadores que guíen y modulen las tensiones entre la proliferación de datos y las limitaciones de la atención, para no naufragar en el sinsentido, canalizando a través de las intervenciones pedagógicas hacia la producción de conocimientos válidos.

Presentación del artículo: 20 de junio de 2015

Fecha de aprobación: 17 de julio de 2015

Fecha de publicación: 30 de octubre de 2015

Apuntes para la revisión teórica de las TIC en el ámbito de la educación superior. Carlos Neri y Diana Fernández Zalazar. 
Neri, C. y Fernández Zalazar, D. (2015). Apuntes para la revisión teórica de las TIC en el ámbito de la educación superior. RED. Revista de Educación a Distancia, 47(3). 30 de Octubre de 2015. Consultado el (dd/mm/aa) en http://www.um.es/ead/red/47

\section{Bibliografía}

Benbenaste, N. (2007). Psicología del mercado y del tipo de sujeto que produce. Buenos Aires: Eudeba.

Bergmann, J., y Sams, A. (2014). Flipped learning: Maximizing face time. $T+D, 68(2)$, 28-31.

Chaiklin, S. y Lave, J. (2001). Estudiar las prácticas. Perspectivas sobre actividad y contexto. Buenos Aires: Amorrortu.

Cid-Sabucedo, A., Pérez-Abellás, A., y Zabalza, M.A. (2009). Las prácticas de enseñanza declaradas de los "mejores profesores" de la Universidad de Vigo. Relieve, 15 (2).

Cole, M. (1999). Psicología Cultural. Una disciplina del pasado y del futuro. Madrid: Morata.

Cole, M. y Engeström, Y. (2001). Enfoque Histórico-cultural de la cognición distribuida. En: G. Salomon (comp.) Cogniciones distribuidas. Consideraciones psicológicas y educativas (pp. 23-74). Buenos Aires: Amorrortu.

Coll, C. (2009). Aprender y enseñar con las TIC: expectativas, realidad y potencialidades en R. Carneiro, J. C. Toscano y T. Díaz (compiladores), Los desafíos de las TIC para el cambio educativo. Madrid: EI-Santillana, Fundación Santillana, 113-126.

Dwyer, D.C., Ringstaff, C., Sandholtz, J.H. (1990). Teacher Beliefs and Practices II: Support for Change (Report No. 9). California: Apple.

Engeström, Y. (2008). The future of activity theory: a rough draft. Conferencia presentada en el Congreso ISCAR (International Society for Cultural and Activity Research). San Diego, 8 al 13 de septiembre de 2008.

Engeström, Y.; Miettinen, R.-L. y Punamaki, R. (1999). Perspectives on Activity Theory. Learning in doing: social, cognitive and computational perspectives. Cambridge: Cambridge University Press.

Hartley, J. (2009). Uses of YouTube- Digital Literacy and the Growth of Knowledge, en Burgess, J. y J. Green, YouTube. OnLine Video and Participatory Culture, Cambridge, UK, Polity Press.

Apuntes para la revisión teórica de las TIC en el ámbito de la educación superior. Carlos Neri y Diana Fernández Zalazar. 
Jonassen. D.H. (1995). Supporting communities of learners with technology: A vision for integrating technology in learning in schools. Educational Technology, 35 (4), $60-62$.

Moreira, M. A. (2003). ¿ Qué aporta Internet al cambio pedagógico en la educación superior?. Universidad de la Laguna.

Morton C. (1996). The modern land of Laputa. Phi Delta Kappan. 77 (6) 416-419.

Neri, C. M., Fernández Zalazar, D. C., Freijo, F., \& Ciacciulli, S. M. (2011). Memorias III Congreso Internacional de Investigación y Práctica Profesional en Psicología, XVIII Jornadas de Investigación, Séptimo Encuentro de Investigadores en Psicología del MERCOSUR 14, 252-255.

Neri, C.; Fernández Zalazar, D. (2008) Telarañas de conocimiento. Educando en tiempos de la Web 2.0. Buenos Aires: Libros y Bytes.

Reig Hernández, D. (2012): Disonancia cognitiva y apropiación de las TIC. Fundación Telefónica Revista Telos 98, 1-2.

Salomon, G. (1992). Las diversas influencias de la tecnología en el desarrollo de la mente. Infancia y Aprendizaje: Journal for the Study of Education and Development (58), 143-159.

Sams, A. (2011). The Flipped Class: Shedding light on the confusion, critique, and hype. Recuperado de: http://www.thedailyriff.com/articles/the-flipped-classshedding-light-on-the-confusion-critique-and-hype-801.php

Salomon, G., Perkins D. N., Globerson T. (2009). Coparticipando en el conocimiento: la ampliación de la inteligencia humana con las tecnologías inteligentes.

Techonologies. Educational Researchen, 20 (3), 2-9.

Sandholtz, J.H., Ringstaff, C., Dwyer, D.C. (1997). Teaching with Technology: Creating Student Centered Classrooms. New York: Teachers College Press.

Santamaría, F. (2010). Evolución y desarrollo de un Entorno Personal de Aprendizaje en la Universidad de León. Digital Education Review, (18), 48-60.

Severance, C., Hardin, J. y Whyte, A. (2008). The comming functionality mash-up in Personal Learning Environments. Interactive Learning Enviroments, 16 (1), 47-62.

Vigotsky, L. S. (2000). El desarrollo de los procesos psicológicos superiores. México: Grijalbo.

Wertsch, J. V. (1988). Vigotsky y la formación social de la mente. Barcelona: Paidós.

Wilson, S., Liber, O., Johnson, M. W., Beauvoir, P., Sharples, P., \& Milligan, C. D. (2007). Personal Learning Environments: Challenging the dominant design of educational systems. Journal of e-Learning and Knowledge Society, 3(2), 27-38.

Apuntes para la revisión teórica de las TIC en el ámbito de la educación superior. Carlos Neri y Diana Fernández Zalazar. 